Bernard P. Cholley

Antoine Vieillard-Baron

Alexandre Mabazaa

\title{
Echocardiography in the ICU: \\ time for widespread use!
}

Published online: 2 March 2006

(C) Springer-Verlag 2006

B. P. Cholley (匹) - A. Mabazaa

Hôpital Lariboisiére, Assistance Publique-Hôpitaux de Paris,

Departement d'Anesthésie-Réanimation,

2 rue Ambroise Paré, 75475 Paris CEDEX 10, France

e-mail: bernard.cholley@1rb.aphp.fr

Tel.: +33-1-49958085

Fax: +33-1-49958073

A. Vieillard-Baron

Hôpital Ambroise Paré, Assistance Publique-Hôpitaux de Paris,

Service de Réanimation Médicale,

9 avenue Charles de Gaulle, 92104 Boulogne, France
The wrong year was given in the citation line. The correct citation line is reproduced here: Intensive Care Med (2006) 32:9-10

DOI 10.1007/s00134-005-2833-8 